\title{
Heterocontrole da fluoretação nos municípios da Região Metropolitana de Curitiba nos anos de 2014 e 2015
}

\author{
External control of fluoridation in the Metropolitan Region \\ of Curitiba in the years of 2014 and 2015
}

\author{
Cristiane Matsuo de Oliveira Piorunneck ${ }^{1}$, Rafael Gomes Ditterich ${ }^{1,2}$, \\ Eliane Carneiro Gomes ${ }^{1,2}$
}

\begin{abstract}
Resumo
Introdução: O heterocontrole, que é o monitoramento da concentração de fluoreto na água de abastecimento, deve ser feito por instituições do Estado e tem sido motivado para garantir a eficácia e a segurança da fluoretação. Este estudo teve como objetivo analisar o heterocontrole de municípios com população acima de 50 mil habitantes da Região Metropolitana de Curitiba nos anos de 2014 e 2015. Metodologia: Dados sobre o heterocontrole foram fornecidos pelos serviços de Vigilância em Saúde dos municípios do estudo e analisados de acordo com o critério técnico do CECOL para a concentração de fluoreto. Resultados: Em alguns municípios e meses, houve falha na realização das coletas de amostras de água em relação ao número adequado para o parâmetro fluoreto. Do total de amostras analisadas, somente $51,5 \%$ estavam na faixa de melhor benefício-risco para a concentração de fluoreto, com $8 \%$ de benefício e risco insignificantes e $0,1 \%$ das amostras com concentração de fluoreto de alto risco, sem nenhum benefício. Conclusão: Os municípios analisados realizaram o heterocontrole, com períodos em que o número de coletas não foi adequado. As concentrações de fluoreto na água de abastecimento dos municípios do estudo apresentaram resultado pouco satisfatório. O heterocontrole é fundamental para manter os teores de fluoreto em níveis adequados.
\end{abstract}

Palavras-chave: fluoretação da água; controle da qualidade da água; abastecimento de água; saúde bucal; cárie dentária.

\begin{abstract}
Introduction: External control is the monitoring of fluoride concentration in the water supply that must be done by state institutions, and has been motivated to ensure the efficacy and safety of fluoridation. This study aimed to analyze the external control of municipalities with more than 50.000 inhabitants in the metropolitan region of Curitiba in the years 2014 and 2015. Methodology: Health surveillance services of the studied municipalities provided external control data, which were analyzed according to the technical criterion of the CECOL for fluoride content. Results: Collection of water samples failed in some municipalities and months for an adequate number for the fluoride parameter. From the total samples analyzed, only $51.5 \%$ were in the best benefit-risk range for the fluoride concentration. Eight percent of the samples presented insignificant benefit and risk and $0.1 \%$ showed a high-risk fluoride concentration, without any benefit. Conclusion: the analyzed municipalities performed the external control, with periods of inadequate sample collections. Fluoride concentrations in the supply water of the study municipalities presented an unsatisfactory result. The main goal of external control is to maintain fluoride levels at adequate levels.
\end{abstract}

Keywords: water fluoridation; water quality control; water supply; oral health; dental caries.

${ }_{1}^{1}$ Programa de Pós-graduação em Saúde Coletiva, Universidade Federal do Paraná (UFPR) - Curitiba (PR), Brasil.

²Departamento de Saúde Comunitária, Universidade Federal do Paraná (UFPR) - Curitiba (PR), Brasil.

Trabalho realizado na Região Metropolitana de Curitiba (PR), Brasil.

Endereço para correspondência: Cristiane Matsuo de Oliveira Piorunneck - Programa de Pós-graduação em Saúde Coletiva, Universidade Federal do Paraná (UFPR), Rua Padre Camargo, 280, $7{ }^{\circ}$ andar - Alto da Glória - CEP: 80060-240 - Curitiba (PR), Brasil - Email: cristianeprefeitura@gmail.com

Fonte de financiamento: recursos próprios.

Conflito de interesses: nada a declarar. 


\section{INTRODUÇÃO}

A fluoretação da água de abastecimento é considerada uma das maiores conquistas da saúde pública no século $\mathrm{XX}^{1}$. Estudos baseados em evidência confirmam essa ação como sendo um método eficaz e socialmente equitativo para oferecer os benefícios preventivos do fluoreto contra a cárie dentária ${ }^{2}$, pois atinge as pessoas independentemente de sua idade e do seu nível educacional e social ${ }^{3}$.

Estima-se que o poder preventivo da fluoretação seja em torno de 40 a $70 \%$ em crianças, reduzindo a perda dentária em adultos de 40 a $60 \%{ }^{4}$. O benefício da fluoretação na prevenção da cárie dentária também está demonstrado em vários estudos ${ }^{5-9}$, e muitas entidades representativas de saúde recomendam a fluoretação, como International Association for Dental Research, European Organization for Caries Research, Organização Pan-Americana de Saúde, Organização Mundial da Saúde e World Dental Federation ${ }^{10}$.

A fluoretação da água de abastecimento começou nos Estados Unidos em 1945. Atualmente, quase $3 / 4$ da população americana tem acesso à água fluoretada, bem como mais de 370 milhões de pessoas em 30 países. Hong Kong e Singapura possuem $100 \%$ de acesso à fluoretação, enquanto a Austrália apresenta uma cobertura da fluoretação em torno de $80 \%$ da população ${ }^{11}$.

No Brasil, a fluoretação teve início em 1954 na cidade de Baixo Guandu, no Espírito Santo ${ }^{12}$, tornando-se medida obrigatória onde exista estação de tratamento de água pela Lei Federal $n^{\circ} 6.050 / 1974^{13}$. A fluoretação da água de abastecimento público constitui um dos elementos norteadores da Política Nacional de Saúde Bucal ${ }^{14}$. De acordo com as diretrizes dessa política, o acesso à água tratada e fluoretada é fundamental para as condições de saúde da população, sendo necessário que políticas públicas garantam a implantação e a ampliação da fluoretação, por ser a forma mais abrangente e socialmente justa de acesso ao fluoreto $^{15}$.

Resultados de levantamentos epidemiológicos de saúde bucal no Brasil mostraram que, após meados dos anos de 1980, houve um expressivo declínio dos indicadores de cárie, com o índice que expressa o número de dentes cariados, perdidos e restaurados com experiência de cárie (CPOD) sendo reduzido de 6,7 no ano de 1986 para 2,8 no ano de 2003, em crianças com 12 anos de idade. Um dos motivos apontados para esses resultados foi a expansão da oferta da água fluoretada ocorrida no período ${ }^{16}$.

Porém, a fluoretação ainda apresenta marcantes desigualdades regionais na sua implantação. Nas regiões Sul e Sudeste, onde se concentra a maior parte da riqueza do país, a implantação da medida avançou, mas foi insuficiente nas regiões Norte e Nordeste ${ }^{17}$. Além da implantação e ampliação da fluoretação no país, é uma prioridade governamental também garantir continuidade e teores adequados de fluoreto na água, com a criação e/ou desenvolvimento de sistemas de vigilância compatíveis por parte dos órgãos de gestão do Sistema Único de Saúde (SUS) ${ }^{15}$.

Estudo de Frazão e Narvai ${ }^{18}$ avaliou a cobertura da fluoretação da água de abastecimento público em municípios brasileiros na primeira década do século XXI, segundo porte demográfico e nível de desenvolvimento humano municipal (IDH-M), e concluiu que a taxa de cobertura populacional da fluoretação aumentou de $67,7 \%$ para $76,3 \%$, com ampliação expressiva em municípios com menos de 10 mil habitantes e com IDH-M baixo ou muito baixo.

Quanto à taxa de cobertura municipal, esse mesmo estudo também mostrou que houve redução da desigualdade, com exceção da desigualdade absoluta entre as categorias de IDH-M. Apesar disso, a política pública da fluoretação atuou produzindo benefícios em saúde, contribuindo para a diminuição de desigualdades e agindo como fator de proteção sanitária no contexto das políticas de proteção social em curso no país ${ }^{18}$.

As concentrações ideais de fluoreto na água são determinadas de acordo com as médias das temperaturas máximas anuais de cada região, ficando o limite de fluoreto estabelecido entre 0,6 a 1,7mg/L pela Portaria do Ministério da Saúde no 635/ $\mathrm{BSB} / 1975^{19}$. Atualmente, a Portaria $\mathrm{n}^{\circ} 2.914 / 2011^{20}$ determina que a concentração máxima permitida de fluoreto na água não deve ultrapassar 1,5mg/L, enquanto a Diretriz Nacional do Plano de Amostragem da Vigilância da Qualidade da Água para Consumo Humano ${ }^{21}$ estabelece parâmetros a serem analisados, como o do fluoreto, e determina competências para o exercício da vigilância da qualidade da água para consumo humano.

No ano de 2009, por iniciativa da Secretaria de Vigilância em Saúde do Ministério da Saúde, foi criado o Centro Colaborador do Ministério da Saúde em Vigilância da Saúde Bucal (CECOL) da Faculdade de Saúde Pública da Universidade de São Paulo (FSP-USP), que tem como responsabilidade a vigilância e a cobertura da fluoretação das águas de abastecimento público como objetivo de suas atividades, por meio do Projeto Vigiflúor. O CECOL, por intermédio de seu corpo de pesquisadores e técnicos, propôs um critério para a classificação das águas segundo o teor de fluoreto e de acordo com a variação da temperatura da região, relacionando as dimensões do benefício da prevenção da cárie dentária pela fluoretação da água de abastecimento e do risco de ocorrer fluorose dentária, pois, com essa classificação de benefício-risco, podem-se esperar interpretações de maior amplitude do que quando se avalia a concentração do fluoreto em apenas duas categorias, "adequada/inadequada"22. A Tabela 1 apresenta esse critério.

A adequação do teor de fluoreto na água de abastecimento é fundamental para a segurança e eficácia preventiva da fluoretação. Se a água estiver com concentração de flúor abaixo do recomendado, não haverá proteção contra a cárie; se a água 
Tabela 1. Relação benefício-risco para localidades onde as médias das temperaturas máximas se situaram abaixo de $26,3^{\circ} \mathrm{C}$

\begin{tabular}{ccc}
$\begin{array}{c}\text { Teor de flúor na } \\
\text { água } \\
(\mathbf{p p m} \text { ou } \mathbf{~ m g} / \mathbf{L})\end{array}$ & $\begin{array}{c}\text { Benefício } \\
\text { (prevenção da } \\
\text { doença cárie) }\end{array}$ & $\begin{array}{c}\text { Risco } \\
\text { (fluorose dentária) }\end{array}$ \\
0,00 a 0,44 & Insignificante & Insignificante \\
0,45 a 0,54 & Mínimo & Baixo \\
0,55 a 0,64 & Moderado & Baixo \\
$\mathbf{0 , 6 5}$ a $\mathbf{0 , 9 4}$ & Máximo & Baixo \\
0,95 a 1,24 & Máximo & Moderado \\
1,25 a 1,44 & Questionável & Alto \\
1,45 ou mais & Malefício & Muito alto \\
\hline
\end{tabular}

Nota: ${ }^{*}$ observa-se que a melhor combinação benefício-risco ocorre na faixa de 0,65 a $0,94 \mathrm{ppm}$. Fonte: CECOL/USP ${ }^{22}$

estiver hiperfluoretada, poderá causar fluorose dentária, que é caracterizada por alterações no processo de mineralização do esmalte dentário ${ }^{14}$.

Para garantir a eficácia preventiva da fluoretação, é indispensável o controle operacional nas estações de tratamento de água, realizado pelas empresas responsáveis pelo processo da fluoretação. Já o monitoramento da fluoretação deve ser realizado pela vigilância sanitária e é conhecido por heterocontrole, sendo definido por Narvai ${ }^{23}$ como o princípio segundo o qual, se um bem ou serviço implicar risco ou representar fator de proteção para a saúde pública, além do controle operacional do responsável pelo processo, deve haver o controle por parte de instituições do Estado.

O heterocontrole tem sido motivado para garantir a eficácia e a segurança da fluoretação ${ }^{24}$, e vários estudos trazem resultados do heterocontrole mostrando variações significativas na concentração de fluoreto em algumas localidades brasileiras ${ }^{25-28}$.

Diante da importância da fluoretação para a saúde pública e da importância do monitoramento das concentrações adequadas de fluoreto na água de abastecimento público, este trabalho teve por objetivo analisar o heterocontrole da fluoretação de municípios com população acima de 50 mil habitantes da Região Metropolitana de Curitiba, no Paraná, nos anos de 2014 e 2015.

\section{MATERIAL E MÉTODOS}

A Região Metropolitana de Curitiba, que é, atualmente, composta por 29 municípios, foi criada no ano de 1973 e é a $8^{\text {a }}$ mais populosa do Brasil, concentrando $30,86 \%$ da população do Estado do Paraná29 . Almirante Tamandaré, Araucária, Campo Largo, Colombo, Fazenda Rio Grande, Pinhais, Piraquara, São José dos Pinhais fazem parte da Região Metropolitana de Curitiba e são os municípios que possuem população acima de 50 mil habitantes. Esse critério de escolha dos municípios seguiu parâmetro estabelecido nos documentos técnicos do $\mathrm{CECOL}^{30}$.

A Tabela 2 mostra a estimativa populacional ${ }^{31}$, a data de início da fluoretação ${ }^{32}$, as Estações de Tratamento de Água (ETA) que
Tabela 2. Número da população, início da fluoretação, Estações de Tratamento de Água (ETAs) que abastecem cada município do estudo e suas Unidades de Tratamento (UTs) correspondentes

\begin{tabular}{|c|c|c|c|c|}
\hline Município & População & $\begin{array}{c}\text { Início da } \\
\text { fluoretação }\end{array}$ & ETAs & UTs \\
\hline \multirow[t]{2}{*}{$\begin{array}{l}\text { Almirante } \\
\text { Tamandaré }\end{array}$} & 114.129 & 1985 & $\begin{array}{l}\text { Iguaçu e } \\
\text { Iraí }\end{array}$ & UT 0014 \\
\hline & & & $\begin{array}{c}\text { Poço } \\
\text { Almirante } \\
\text { Tamandaré }\end{array}$ & UT 0341 \\
\hline \multirow{4}{*}{$\begin{array}{c}\text { Araucária } \\
\text { Campo } \\
\text { Largo }\end{array}$} & 135.459 & 1984 & Passaúna & UT 0012 \\
\hline & 125.719 & 1984 & $\begin{array}{c}\text { Campo } \\
\text { Largo e Rio } \\
\text { Verde }\end{array}$ & UT 0121 \\
\hline & & & $\begin{array}{c}\text { Poço } \\
\text { Rebouças }\end{array}$ & UT 0122 \\
\hline & & & Rio Verde & UT 0123 \\
\hline Colombo & 234.941 & 1988 & $\begin{array}{l}\text { Palmital } \\
\text { Karst }\end{array}$ & $\begin{array}{l}\text { UT } 0791 \\
\text { UT } 0792\end{array}$ \\
\hline $\begin{array}{l}\text { Fazenda } \\
\text { Rio }\end{array}$ & 93.730 & 1993 & $\begin{array}{l}\text { Passaúna e } \\
\text { Miringuava }\end{array}$ & UT 0015 \\
\hline Grande & & & Despique & UT 3271 \\
\hline Pinhais & 128.256 & 1978 & Iraí & UT 0013 \\
\hline Piraquara & 106.132 & 1985 & $\begin{array}{l}\text { Iguaçu } \\
\text { Iraí }\end{array}$ & $\begin{array}{l}\text { UT } 0011 \\
\text { UT } 0013\end{array}$ \\
\hline \multirow[t]{4}{*}{$\begin{array}{c}\text { São } \\
\text { José dos } \\
\text { Pinhais }\end{array}$} & 302.759 & 1974 & $\begin{array}{l}\text { Iguaçu } \\
\text { Rio } \\
\text { Pequeno }\end{array}$ & $\begin{array}{l}\text { UT } 0011 \\
\text { UT } 0251\end{array}$ \\
\hline & & & Miringuava & UT 0252 \\
\hline & & & $\begin{array}{c}\text { Poços da } \\
\text { Borda do } \\
\text { Campo }\end{array}$ & UT 0253 \\
\hline & & & Cotia & UT 0254 \\
\hline
\end{tabular}

Fonte: Elaborada pela autora com base em $\mathrm{IBGE}^{31}, \mathrm{CECOL}^{32}$ e dados fornecidos pela SANEPAR (ano 2016)

abastecem cada município e as Unidades de Tratamento (UT). As UTs utilizam códigos especialmente criados pela Companhia de Saneamento do Paraná (SANEPAR), responsável pelo abastecimento público e pela fluoretação da região estudada, para identificar a origem das águas, ou seja, de quais ETAs a água se origina, pois, em alguns pontos, as águas provenientes das ETAs se misturam; assim, com os códigos criados, é possível identificar a origem correta da água.

Os dados obtidos sobre o monitoramento das concentrações de fluoreto na água de abastecimento foram fornecidos pelos Departamentos de Vigilância em Saúde dos municípios do estudo, que são os órgãos responsáveis pelo heterocontrole. Posteriormente, os dados foram tabulados no programa Excel 2010 e analisados pelo programa estatístico SPSS 21, de acordo com o critério preconizado pelo $\mathrm{CECOL}^{22}$ para a concentração de fluoreto, conforme Tabela 1. Os municípios do estudo apresentaram média de temperatura anual abaixo de $26^{\circ} \mathrm{C}^{33-40}$. O valor da média de temperatura da região foi utilizado para a análise da concentração de fluoreto na água de abastecimento. 
Foi utilizado o teste qui-quadrado com nível de significância de $5 \%$ e aplicado o teste de diferença de proporções para a análise da variação da concentração de fluoreto nos municípios nos anos de 2014 e 2015. Os municípios estão codificados e não serão identificados na apresentação dos resultados. Este trabalho foi aprovado pelo Comitê de Ética em Pesquisa (CEP/SD) da Universidade Federal do Paraná, CAAE 51737115.8.0000.0102, Parecer nº 1.474.946.

\section{RESULTADOS}

A Tabela 3 mostra o número de amostras de água obtidas por mês pelos serviços de Vigilância em Saúde de cada município do estudo para o parâmetro fluoreto nos anos de 2014 e 2015, comparados com o número mínimo de amostras de água recomendado pela Diretriz Nacional do Plano de Amostragem da Vigilância da Qualidade da Água para Consumo Humano ${ }^{21}$

Tabela 3. Número de amostras de água obtidas pela Vigilância em Saúde dos municípios estudados por mês, nos anos de 2014 e 2015 , e o número de amostras recomendada pela Diretriz Nacional do Plano de Amostragem da Vigilância da Qualidade da Água para Consumo Humano

\begin{tabular}{|c|c|c|c|c|c|c|c|c|c|c|}
\hline \multirow{2}{*}{ MÊS } & \multirow{2}{*}{ ANO } & \multicolumn{8}{|c|}{ MUNICÍPIO } & \multirow{2}{*}{ TOTAL } \\
\hline & & 1 & 2 & 3 & 4 & 5 & 6 & 7 & 8 & \\
\hline \multirow[t]{3}{*}{ Janeiro } & 2014 & $0(9)$ & $0(9)$ & $0(9)$ & $5(13)$ & $4(7)$ & $86(9)$ & $3(9)$ & $22(13)$ & $120(78)$ \\
\hline & 2015 & $17(9)$ & $7(9)$ & $21(9)$ & $20(13)$ & $0(7)$ & $20(9)$ & $18(9)$ & $12(13)$ & $115(78)$ \\
\hline & Total & $17(18)$ & $7(18)$ & $21(18)$ & $25(26)$ & $4(14)$ & $106(18)$ & $21(18)$ & $34(26)$ & $235(156)$ \\
\hline \multirow[t]{3}{*}{ Fevereiro } & 2014 & 7 (9) & $0(9)$ & $0(9)$ & $10(13)$ & $4(7)$ & $71(9)$ & $9(9)$ & $28(13)$ & $129(78)$ \\
\hline & 2015 & $6(9)$ & $10(9)$ & $12(9)$ & $17(13)$ & $15(7)$ & $15(9)$ & $0(9)$ & $18(13)$ & $93(78)$ \\
\hline & Total & $13(18)$ & $10(18)$ & $12(18)$ & $27(26)$ & $19(14)$ & $86(18)$ & $9(18)$ & $46(26)$ & $222(156)$ \\
\hline \multirow[t]{3}{*}{ Março } & 2014 & 7 (9) & $0(9)$ & $0(9)$ & $13(13)$ & $8(7)$ & $15(9)$ & $5(9)$ & $9(13)$ & $57(78)$ \\
\hline & 2015 & $20(9)$ & $16(9)$ & $26(9)$ & $30(13)$ & $15(7)$ & $15(9)$ & $23(9)$ & $29(13)$ & $174(78)$ \\
\hline & Total & $27(18)$ & $16(18)$ & $26(18)$ & $43(26)$ & $23(14)$ & $30(18)$ & $28(18)$ & $38(26)$ & $231(156)$ \\
\hline \multirow[t]{3}{*}{ Abril } & 2014 & $3(9)$ & $0(9)$ & $0(9)$ & $9(13)$ & $4(7)$ & $10(9)$ & $1(9)$ & $10(13)$ & $37(78)$ \\
\hline & 2015 & $14(9)$ & $7(9)$ & $15(9)$ & $27(13)$ & $15(7)$ & $18(9)$ & $11(9)$ & $38(13)$ & $145(78)$ \\
\hline & Total & $17(18)$ & $7(18)$ & $15(18)$ & $36(26)$ & $19(14)$ & $28(18)$ & $12(18)$ & $48(26)$ & $182(156)$ \\
\hline \multirow[t]{3}{*}{ Maio } & 2014 & $4(9)$ & $0(9)$ & $0(9)$ & $15(13)$ & $4(7)$ & $19(9)$ & $15(9)$ & $8(13)$ & $65(78)$ \\
\hline & 2015 & $21(9)$ & $20(9)$ & $21(9)$ & $22(13)$ & $15(7)$ & $24(9)$ & $21(9)$ & $42(13)$ & $186(78)$ \\
\hline & Total & $25(18)$ & $20(18)$ & $21(18)$ & $37(26)$ & $19(14)$ & $43(18)$ & $36(18)$ & $50(26)$ & $251(156)$ \\
\hline \multirow[t]{3}{*}{ Junho } & 2014 & $8(9)$ & $0(9)$ & $0(9)$ & $20(13)$ & $8(7)$ & $10(9)$ & $19(9)$ & $12(13)$ & 77 (78) \\
\hline & 2015 & $19(9)$ & $7(9)$ & $25(9)$ & $42(13)$ & $15(7)$ & $27(9)$ & $20(9)$ & $28(13)$ & $183(78)$ \\
\hline & Total & 27 (18) & $7(18)$ & 25 (18) & $62(26)$ & $23(14)$ & 37 (18) & 39 (18) & $40(26)$ & $260(156)$ \\
\hline \multirow[t]{3}{*}{ Julho } & 2014 & $8(9)$ & $25(9)$ & $10(9)$ & $0(13)$ & $6(7)$ & $20(9)$ & $16(9)$ & $8(13)$ & $93(78)$ \\
\hline & 2015 & $13(9)$ & $17(9)$ & $15(9)$ & $28(13)$ & $10(7)$ & $25(9)$ & $0(9)$ & $18(13)$ & $126(78)$ \\
\hline & Total & $21(18)$ & $42(18)$ & $25(18)$ & $28(26)$ & $16(14)$ & $45(18)$ & $16(18)$ & $26(26)$ & $21(156)$ \\
\hline \multirow[t]{3}{*}{ Agosto } & 2014 & $8(9)$ & $5(9)$ & $16(9)$ & $0(13)$ & $8(7)$ & $27(9)$ & $16(9)$ & $4(13)$ & $84(78)$ \\
\hline & 2015 & $20(9)$ & $11(9)$ & $20(9)$ & $28(13)$ & $15(7)$ & $23(9)$ & $0(9)$ & $29(13)$ & $146(78)$ \\
\hline & Total & $28(18)$ & $16(18)$ & $36(18)$ & $28(26)$ & $23(14)$ & $50(18)$ & $16(18)$ & $33(26)$ & $230(156)$ \\
\hline \multirow[t]{3}{*}{ Setembro } & 2014 & $8(9)$ & $0(9)$ & $15(9)$ & $0(13)$ & $8(7)$ & $15(9)$ & $16(9)$ & $23(13)$ & $85(78)$ \\
\hline & 2015 & $20(9)$ & $9(9)$ & $25(9)$ & $17(13)$ & $17(7)$ & $30(9)$ & $0(9)$ & $18(13)$ & $136(78)$ \\
\hline & Total & $28(18)$ & $9(18)$ & $40(18)$ & $17(26)$ & $25(14)$ & $45(18)$ & $16(18)$ & $41(26)$ & $221(156)$ \\
\hline \multirow[t]{3}{*}{ Outubro } & 2014 & $8(9)$ & $34(9)$ & $4(9)$ & $0(13)$ & $8(7)$ & $28(9)$ & $12(9)$ & $53(13)$ & $147(78)$ \\
\hline & 2015 & $14(9)$ & $9(9)$ & $15(9)$ & $18(13)$ & $12(7)$ & $34(9)$ & $21(9)$ & $18(13)$ & $141(78)$ \\
\hline & Total & $22(18)$ & $4(18)$ & $19(18)$ & $18(26)$ & $20(14)$ & $62(18)$ & $33(18)$ & $71(26)$ & $288(156)$ \\
\hline \multirow[t]{3}{*}{ Novembro } & 2014 & $8(9)$ & $0(9)$ & $0(9)$ & $0(13)$ & $8(7)$ & $22(9)$ & $15(9)$ & $65(13)$ & $118(78)$ \\
\hline & 2015 & $16(9)$ & $10(9)$ & $20(9)$ & $12(13)$ & $24(7)$ & $35(9)$ & $27(9)$ & $26(13)$ & $170(78)$ \\
\hline & Total & 24 (18) & $10(18)$ & $20(18)$ & $12(26)$ & $32(14)$ & $57(18)$ & $42(18)$ & $91(26)$ & $288(156)$ \\
\hline \multirow[t]{3}{*}{ Dezembro } & 2014 & $16(9)$ & $0(9)$ & $0(9)$ & $0(13)$ & $0(7)$ & $12(9)$ & $5(9)$ & $18(13)$ & $51(78)$ \\
\hline & 2015 & $7(9)$ & $6(9)$ & $10(9)$ & 34 (13) & $5(7)$ & $35(9)$ & $10(9)$ & $12(13)$ & $119(78)$ \\
\hline & Total & $23(18)$ & $6(18)$ & $10(18)$ & $34(26)$ & $5(14)$ & 47 (18) & $15(18)$ & $30(26)$ & $170(156)$ \\
\hline \multirow[t]{3}{*}{ TOTAL } & 2014 & $8(108)$ & $64(108)$ & 45 (108) & $72(156)$ & $70(84)$ & 335 (108) & $132(108)$ & $260(156)$ & $1063(936)$ \\
\hline & 2015 & $187(108)$ & $129(108)$ & $225(108)$ & $295(156)$ & $158(84)$ & 301 (108) & $151(108)$ & $288(156)$ & $1734(936)$ \\
\hline & Total & $272(216)$ & $193(216)$ & $270(216)$ & $367(312)$ & $228(168)$ & $636(216)$ & $283(216)$ & $548(312)$ & $2797(1872)$ \\
\hline
\end{tabular}

Nota: Os dados entre parênteses correspondem ao número mínimo de amostras recomendado por mês pela Diretriz Nacional do Plano de Amostragem da Vigilância da Qualidade da Água para Consumo Humano. Os dados em negrito são dos municípios e meses em que não foi alcançado o número mínimo de amostras. Os dados sublinhados são daqueles que não obtiveram nenhuma amostra. Fonte: Elaborada pela autora com base em dados da Vigilância em Saúde dos municípios participantes do estudo (ano 2015) e Diretriz Nacional do Plano de Amostragem da Vigilância da Qualidade da Água para Consumo Humano ${ }^{21}$ 
para esse mesmo parâmetro, que é determinado segundo a faixa populacional do município.

No caso dos municípios analisados neste estudo, para uma população de 50 mil a 100 mil habitantes, são necessárias no mínimo 7 amostras; de 100.001 a 200 mil habitantes são 9 amostras; e de 200.001 a 500 mil habitantes são no mínimo 13 amostras mensais para o monitoramento da concentração de flúor na água de abastecimento público ${ }^{21}$.

Os serviços de Vigilância em Saúde dos municípios estudados obtiveram, no ano de 2014, 1.063 amostras de água para o parâmetro fluoreto e, no ano de 2015, 1.734 amostras, totalizando, nesses dois anos, 2.797 amostras de água. Esses números superam a perspectiva da soma de amostras mínimas recomendadas por mês, quando se analisa a população de cada município no período de cada ano. Porém, foi possível observar, em alguns municípios e em alguns meses nos anos de 2014 e 2015, um número de amostras abaixo do mínimo recomendado, bem como, em alguns municípios, certos meses em que nenhuma amostra de água para o parâmetro fluoreto foi coletada.

A Tabela 4 mostra os resultados encontrados de acordo com o critério do consenso técnico do $\mathrm{CECOL}^{22}$ para temperaturas abaixo de $26^{\circ} \mathrm{C}$, dos oito municípios participantes do estudo, nos anos de 2014 e 2015. O teste qui-quadrado com nível de significância de 5\% mostrou que existe dependência entre os níveis de fluoreto e os municípios analisados, ou seja, em alguns estratos da variação de fluoreto, há diferença estatística entre os municípios.

O teste de diferença entre proporções mostrou onde estão essas diferenças por meio das letras sobrescritas na Tabela 4, em que: letras iguais mostram municípios com porcentagem

Tabela 4. Resultados obtidos da concentração de fluoreto de acordo com critério técnico do Centro Colaborador do Ministério da Saúde em Vigilância da Saúde Bucal (CECOL) por município, 2014 a 2015

\begin{tabular}{|c|c|c|c|c|c|c|c|c|c|c|}
\hline \multirow{2}{*}{\multicolumn{2}{|c|}{ CONCENTRAÇÃO DE FLÚOR }} & \multicolumn{8}{|c|}{ Município } & \multirow{2}{*}{ TOTAL } \\
\hline & & 1 & 2 & 3 & 4 & 5 & 6 & 7 & 8 & \\
\hline \multirow[t]{3}{*}{0,00 a 0,44} & Contagem & $21_{\mathrm{a}, \mathrm{b}, \mathrm{c}, \mathrm{d}, \mathrm{e}}$ & $72_{\mathrm{f}}$ & $8_{\mathrm{d}, \mathrm{e}}$ & $17_{c, \mathrm{e}}$ & $29_{\mathrm{b}}$ & $32_{\mathrm{a}, \mathrm{c}, \mathrm{d}, \mathrm{e}}$ & $10_{\mathrm{a}, \mathrm{c}, \mathrm{d}, \mathrm{e}}$ & $34_{\mathrm{a}, \mathrm{b}, \mathrm{c}, \mathrm{d}, \mathrm{e}}$ & 223 \\
\hline & $\begin{array}{l}\% \text { dentro de } \\
\text { município }\end{array}$ & $7,7 \%$ & $37,3 \%$ & $3,0 \%$ & $4,6 \%$ & $12,7 \%$ & $5,0 \%$ & $3,5 \%$ & $6,2 \%$ & $8,0 \%$ \\
\hline & $\%$ do total & $0,8 \%$ & $2,6 \%$ & $0,3 \%$ & $0,6 \%$ & $1,0 \%$ & $1,1 \%$ & $0,4 \%$ & $1,2 \%$ & $8,0 \%$ \\
\hline \multirow[t]{3}{*}{0,45 a 0,54} & Contagem & 34 & 23 & 30 & $32_{\mathrm{a}}$ & $32 \mathrm{a}$ & $51_{a}$ & 34 & $41_{a}$ & 277 \\
\hline & $\begin{array}{l}\text { \% dentro de } \\
\text { município }\end{array}$ & $12,5 \%$ & $11,9 \%$ & $11,1 \%$ & $8,7 \%$ & $14,0 \%$ & $8,0 \%$ & $12,0 \%$ & $7,5 \%$ & $9,9 \%$ \\
\hline & $\%$ do total & $1,2 \%$ & $0,8 \%$ & $1,1 \%$ & $1,1 \%$ & $1,1 \%$ & $1,8 \%$ & $1,2 \%$ & $1,5 \%$ & $9,9 \%$ \\
\hline \multirow[t]{3}{*}{0,55 a 0,64} & Contagem & $85_{a}$ & $37_{\mathrm{a}, \mathrm{b}}$ & $62_{\mathrm{a}, \mathrm{b}}$ & $58_{\mathrm{b}}$ & $57_{\mathrm{a}, \mathrm{b}}$ & $140_{\mathrm{a}, \mathrm{b}}$ & $66_{\mathrm{a}, \mathrm{b}}$ & $118_{\mathrm{a}, \mathrm{b}}$ & 623 \\
\hline & $\begin{array}{l}\text { \% dentro de } \\
\text { município }\end{array}$ & $31,3 \%$ & $19,2 \%$ & $23,0 \%$ & $15,8 \%$ & $25,0 \%$ & $22,0 \%$ & $23,3 \%$ & $21,5 \%$ & $22,3 \%$ \\
\hline & $\%$ do total & $3,0 \%$ & $1,3 \%$ & $2,2 \%$ & $2,1 \%$ & $2,0 \%$ & $5,0 \%$ & $2,4 \%$ & $4,2 \%$ & $22,3 \%$ \\
\hline \multirow[t]{3}{*}{0,65 a 0,94} & Contagem & $122_{\mathrm{a}}$ & $55_{b}$ & $155_{\mathrm{a}, \mathrm{c}}$ & $231_{c}$ & $102_{\mathrm{a}}$ & $342_{a, c}$ & $130_{\mathrm{a}}$ & $304_{a, c}$ & 1441 \\
\hline & $\begin{array}{l}\% \text { dentro de } \\
\text { município }\end{array}$ & $44,9 \%$ & $28,5 \%$ & $57,4 \%$ & $62,9 \%$ & $44,7 \%$ & $53,8 \%$ & $45,9 \%$ & $55,5 \%$ & $51,5 \%$ \\
\hline & $\%$ do total & $4,4 \%$ & $2,0 \%$ & $5,5 \%$ & $8,3 \%$ & $3,6 \%$ & $12,2 \%$ & $4,6 \%$ & $10,9 \%$ & $51,5 \%$ \\
\hline \multirow[t]{3}{*}{0,95 a 1,24} & Contagem & $10_{a}$ & $6_{a, b}$ & $15_{\mathrm{a}, \mathrm{b}}$ & $28_{a, b, c}$ & $8_{\mathrm{a}, \mathrm{b}}$ & $64_{b, c}$ & $43_{c}$ & $48_{\mathrm{a}, \mathrm{b}, \mathrm{c}}$ & 222 \\
\hline & $\begin{array}{l}\% \text { dentro de } \\
\text { município }\end{array}$ & $3,7 \%$ & $3,1 \%$ & $5,6 \%$ & $7,6 \%$ & $3,5 \%$ & $10,1 \%$ & $15,2 \%$ & $8,8 \%$ & $7,9 \%$ \\
\hline & $\%$ do total & $0,4 \%$ & $0,2 \%$ & $0,5 \%$ & $1,0 \%$ & $0,3 \%$ & $2,3 \%$ & $1,5 \%$ & $1,7 \%$ & $7,9 \%$ \\
\hline \multirow[t]{3}{*}{1,25 a 1,44} & Contagem & $0_{a}$ & $0_{a}$ & $0_{a}$ & $0_{a}$ & $0_{a}$ & $5_{a}$ & $0_{a}$ & $3_{\mathrm{a}}$ & 8 \\
\hline & $\begin{array}{l}\% \text { dentro de } \\
\text { município }\end{array}$ & $0,0 \%$ & $0,0 \%$ & $0,0 \%$ & $0,0 \%$ & $0,0 \%$ & $0,8 \%$ & $0,0 \%$ & $0,5 \%$ & $0,3 \%$ \\
\hline & $\%$ do total & $0,0 \%$ & $0,0 \%$ & $0,0 \%$ & $0,0 \%$ & $0,0 \%$ & $0,2 \%$ & $0,0 \%$ & $0,1 \%$ & $0,3 \%$ \\
\hline \multirow[t]{3}{*}{1,45 ou mais } & Contagem & $0_{a}$ & $0_{a}$ & $0_{a}$ & $1_{a}$ & $0_{a}$ & $2 \mathrm{a}$ & $0_{a}$ & $0_{a}$ & 3 \\
\hline & $\begin{array}{l}\text { \% dentro de } \\
\text { município }\end{array}$ & $0,0 \%$ & $0,0 \%$ & $0,0 \%$ & $0,3 \%$ & $0,0 \%$ & $0,3 \%$ & $0,0 \%$ & $0,0 \%$ & $0,1 \%$ \\
\hline & $\%$ do total & $0,0 \%$ & $0,0 \%$ & $0,0 \%$ & $0,0 \%$ & $0,0 \%$ & $0,1 \%$ & $0,0 \%$ & $0,0 \%$ & $0,1 \%$ \\
\hline \multirow{3}{*}{$\begin{array}{l}\text { TOTAL } \\
\text { \% dentro de } \\
\text { município } \\
\% \text { do total }\end{array}$} & Contagem & 272 & 193 & 270 & 367 & 228 & 636 & 283 & 548 & \multirow[t]{3}{*}{2797} \\
\hline & $100,0 \%$ & $100,0 \%$ & $100,0 \%$ & $100,0 \%$ & $100,0 \%$ & $100,0 \%$ & $100,0 \%$ & $100,0 \%$ & $100,0 \%$ & \\
\hline & $9,7 \%$ & $6,9 \%$ & $9,7 \%$ & $13,1 \%$ & $8,2 \%$ & $22,7 \%$ & $10,1 \%$ & $19,6 \%$ & $100,0 \%$ & \\
\hline
\end{tabular}

Nota: Contagem=número de coletas de amostras de água realizadas em cada categoria de concentração de fluoreto. Cada letra inscrita denota um subconjunto de municípios cujas proporções da coluna não diferem significativamente entre si no nível 0,05. Concentração de fluoreto entre 0,65 a 0,94ppm=máximo benefício e baixo risco. Fonte: Vigilância em Saúde dos municípios participantes do estudo (ano 2015) 
igual de concentração de fluoreto dentro de uma mesma faixa de variação e letras diferentes mostram municípios com porcentagens estatisticamente diferentes entre si dentro de determinada faixa de variação de fluoreto.

A variação da concentração de fluoreto entre 0,00 e 0,44 , que representa benefício insignificante para a prevenção da cárie e risco insignificante de produzir fluorose dentária, totalizou $8 \%$ das amostras coletadas no geral. Entre os oito municípios analisados, somente o município 2 foi o que estatisticamente diferiu dos demais, com uma porcentagem de 37,3\% das amostras coletadas dentro do município.

Entre 0,45 e 0,54 de concentração de flúor, que representa benefício considerado mínimo e risco baixo, houve $9,9 \%$ das amostras coletadas no geral, sem nenhum município diferindo estatisticamente entre si. O teor de flúor entre 0,55 e 0,64, que tem benefício moderado e baixo risco, apontou $22,3 \%$ do total das amostras, com os municípios 1 e município 4 diferindo entre si, com, respectivamente, 31,3 e 15,8\% das amostras coletadas dentro do município.

A faixa de concentração de fluoreto entre 0,65 e 0,94 , que é considerada a de melhor combinação benefício-risco, com um máximo benefício para a prevenção da cárie e baixo risco de produzir fluorose dentária, apresentou $51,5 \%$ do total de amostras coletadas, sendo o município 2 o que diferiu estatisticamente dos demais, com uma porcentagem de somente $28,5 \%$ das amostras coletadas dentro do município nessa faixa de concentração de fluoreto.

Os valores de concentração de fluoreto entre 0,95 e 1,24ppm, que já representa risco moderado para produzir fluorose dentária, e entre 1,25 e 1,44, que tem o benefício questionável e um alto risco para fluorose, totalizaram, respectivamente, 7,9 e 3\% das amostras coletadas no geral, com nenhum município diferindo estatisticamente entre si. Já o teor de fluoreto de 1,45 ou mais, que apresenta malefício e risco muito alto de fluorose, representou somente $0,1 \%$ das amostras, também com nenhum município diferindo estatisticamente entre si.

\section{DISCUSSÃO}

Os municípios do estudo apresentam a fluoretação das águas de abastecimento público como benefício há mais de 20 anos, fato que colabora com a afirmação de estudos que colocam a região Sul como uma das melhores em cobertura de fluoretação no Brasil, com valores em torno de $88 \%{ }^{41}$. Porém, mesmo após tantos anos de fluoretação das águas, ainda foram percebidas dificuldades na maioria desses municípios com relação ao procedimento básico do heterocontrole, que é realizar o número mínimo de coletas e a análise de amostras de água para o parâmetro fluoreto por mês; inclusive, em alguns meses, observou-se que nenhuma coleta e análise do parâmetro fluoreto foi realizada no período do estudo.
Essa situação aconteceu, principalmente, no ano de 2014, com uma melhora no número de coletas realizadas no ano de 2015. Os municípios justificaram a dificuldade na coleta de amostras de água para o parâmetro fluoreto no ano de 2014 a fatores relacionados às análises laboratoriais das amostras, ou seja, à falta de cotas do laboratório do Estado ou a dificuldades na contratação de laboratórios particulares para as análises, situações que os municípios buscaram regularizar no ano de 2015, mostrando a preocupação dos serviços de Vigilância em Saúde dos municípios para o aprimoramento no processo do heterocontrole.

A Diretriz Nacional do Plano de Amostragem ${ }^{21}$ visa orientar os municípios na elaboração e implementação dos planos de amostragem da vigilância da qualidade da água para consumo humano no que diz respeito ao quantitativo mínimo de amostras, à frequência de amostragem, aos parâmetros que devem ser analisados, por exemplo, o fluoreto, e às orientações para a seleção dos pontos de coleta. Também o $\mathrm{CECOL}^{30}$ elaborou um manual com o objetivo de orientar os procedimentos para identificação dos pontos de amostragem para a coleta de água.

Portanto, além do número mínimo de coletas de amostras de água que devem ser realizadas, é fundamental que os municípios realizem um correto plano de amostragem, levando em consideração e observando com atenção todos os critérios propostos nas diretrizes ou em outros documentos, como o do CECOL, para que, dessa maneira, o heterocontrole seja mais efetivo.

Com relação aos resultados da concentração de fluoreto na água de abastecimento, observou-se, neste estudo, que somente $51,5 \%$ das amostras coletadas nesses municípios encontraram-se dentro da faixa de concentração de fluoreto que representa a melhor combinação benefício-risco nos anos de 2014 e 2015; os $40,2 \%$ estavam na faixa abaixo da considerada de melhor combinação benefício-risco; e os outros $8,3 \%$ situavam-se na faixa acima da considerada de melhor combinação benefício-risco.

Alguns outros estudos no Brasil também utilizaram o critério técnico do CECOL para avaliação da concentração de fluoreto na água e mostraram resultados em que a concentração de fluoreto apresentou variações. O estudo com resultado mais aproximado ao encontrado nesta pesquisa foi realizado no município de São José do Rio Preto, São Paulo, entre 2003 e 2011, que encontrou $56 \%$ das amostras na combinação de benefício máximo e risco baixo ${ }^{42}$. No entanto, esse mesmo estudo mostrou que somente $32 \%$ das amostras estavam na categoria de benefício máximo e risco baixo na cidade de Nova Aliança, também em São Paulo.

Outros estudos também mostraram porcentagens de fluoreto com resultados pouco satisfatórios, como no município de São Luís, no Maranhão, entre os anos de 2008 e 2009, em que 48,3\% das amostras foram consideradas inadequadas de acordo com o critério técnico do $\mathrm{CECOL}^{43}$. No estudo da cidade de Nova 
Friburgo, no Rio de Janeiro, entre os anos de 2010 e 2011, somente $48,6 \%$ das amostras estavam dentro da faixa considerada de melhor combinação benefício-risco, com 51,4\% abaixo de 0,65ppmF e nenhuma amostra acima de 0,94ppmF ${ }^{44}$.

Algumas pesquisas apresentaram melhores resultados na porcentagem da concentração de fluoreto, como a realizada no município de Jaguaribara, no Ceará, entre 2010 e 2011, que mostrou $63,9 \%$ das amostras na melhor faixa de benefício-risco e $25 \%$ apresentando risco e benefícios insignificantes em relação à fluorose dentária e à prevenção da cárie ${ }^{45}$. Também em Araçatuba, no interior de São Paulo, entre os anos de 2004 e 2012 , uma pesquisa mostrou que $67,2 \%$ das amostras coletadas ofereciam a melhor combinação benefício-risco, ao passo que as amostras com benefício e risco insignificantes foram de 3,8\%, e as de risco muito alto, apenas $0,5 \%{ }^{46}$.

O melhor resultado foi o do estudo realizado na cidade de Maringá, no Paraná, no ano de 2010, que mostrou que $86 \%$ das amostras coletadas do sistema de abastecimento de água estavam de acordo com a melhor combinação benefício-risco ${ }^{47}$.

De acordo com o CECOL, se variações nas concentrações de fluoreto ocorrerem somente de maneira esporádica, podem não representar prejuízo para a fluoretação. Por exemplo, concentrações de fluoreto de benefício insignificante ou risco muito alto são toleráveis desde que aconteçam esporadicamente por um dia ao longo dos meses de um ano. Concentrações de benefício mínimo ou risco alto são aceitáveis apenas se não forem constantes por mais do que sete dias ao longo dos meses do ano; já concentrações de benefício ou risco moderado são toleráveis somente se não forem constantes por mais do que 21 dias durante o período de um $\mathrm{ano}^{22}$.
Ainda com relação aos resultados encontrados nas concentrações de fluoreto nos municípios, de acordo com o critério do consenso técnico do CECOL, o município 2 destacou-se dos demais por apresentar concentração de fluoreto de apenas 28,5\% dentro da faixa considerada de melhor combinação benefício-risco, sendo estatisticamente relevante essa diferença com relação a essa concentração de fluoreto encontrada nos outros municípios.

Essa diferença deve ser investigada pelo serviço de Vigilância em Saúde do município 2, comparando esses resultados com os do controle operacional realizado pela empresa responsável pela fluoretação, para que sejam identificadas as circunstâncias das inconformidades na concentração de fluoreto na água de abastecimento público e, se necessário, sejam tomadas providências para manter as concentrações de fluoreto em níveis seguros e efetivos para benefício da população.

\section{CONCLUSÃO}

De acordo com os resultados apresentados no estudo, pode-se concluir que os municípios analisados realizaram o heterocontrole, porém apresentaram períodos em que o número de coletas não foi adequado, o que pode comprometer todo o processo de vigilância.

A concentração de fluoreto na água de abastecimento dos municípios do estudo apresentou resultado pouco satisfatório, com $51 \%$ das amostras no nível de melhor benefício-risco e ainda um dos municípios com somente 28,5\% dentro dessa mesma categorização.

O heterocontrole deve ser realizado de maneira sistemática e contínua, pois é fundamental para manter os teores de fluoreto em níveis adequados, garantindo a qualidade da fluoretação e o benefício da prevenção da cárie dentária para a população.

\section{REFERÊNCIAS}

1. Centers for Disease Control and Prevention. Ten great public health achievements in the 20th century. Atlanta; 2013 [citado em 2017 maio 31]. Disponível em: https://www.cdc.gov/about/history/tengpha.htm

2. Yeung CA. A systematic review of the efficacy and safety of fluoridation. Evid Based Dent. 2008;9(2):39-43. http://dx.doi.org/10.1038/sj.ebd.6400578. PMid:18584000.

3. U.S. Department of Health and Human Services Federal Panel on Community Water Fluoridation. U.S Public Health Service recommendation for fluoride concentration in drinking water for the prevention os dental caries. Public Health Rep. 2015 jul-ago;130(4):318-31

4. Centers for Disease Control and Prevention. Achievements in public health,1900-1999: fluoridation of drinking water to prevent dental caries. Morb Mortal Wkly Rep. 1999;48(4):993-6. PMid:10577493.

5. Armfield JM. Community effectiveness of public water fluoridation in reducing children's dental disease. Public Health Rep. 2010;125(5):655-64. http://dx.doi.org/10.1177/003335491012500507. PMid:20873281.
6. Frazão P, Peres MA, Cury JA. Qualidade da água para consumo humano e concentração de fluoreto. Rev Saude Publica. 2011;45(5):964-73. http:// dx.doi.org/10.1590/S0034-89102011005000046. PMid:21789449.

7. Narvai PC, Frias AC, Fratucci MVB, Antunes JLF, Carnut L, Frazão P. Fluoretação da água em capitais brasileiras no início do século XXI: a efetividade em questão. Saúde Debate. 2014;38:562-71.

8. Blinkhorn AS, Byun R, Johnson G, Metha P, Kay M, Lewis P. The Dental Health of primary school children living in fluoridated, pre-fluoridated and non-fluoridated communities in New South Wales, Australia. BMC Oral Health. 2015;15(1):9. http://dx.doi.org/10.1186/1472-6831-15-9. PMid:25604625.

9. Peres MA, Peres KG, Barbato PR, Höfelmann DA. Access to fluoridated water and adult dental caries. J Dent Res. 2016;95(8):868-74. http://dx.doi. org/10.1177/0022034516643064. PMid:27053119.

10. Buzalaf MAR, Moraes CM, Olympio KPK, Pessan JP, Grizzo LT, Silva TL, et al. Seven years of external control of fluoride levels in the public 
water supply in Bauru, São Paulo, Brazil. J Appl Oral Sci. 2013;21(1):92-8. http://dx.doi.org/10.1590/1678-7757201302196. PMid:23559119.

11. Howat P, Binns C, Jancey J. New international review supports community water fluoridation as an effective and safe dental health promotion measure. Health Promot J Austr. 2015;26(1):1-3. http://dx.doi.org/10.1071/ HEv26n1_ED. PMid:26149252.

12. Pucca GA Jr, Costa JFR, Chagas LD, Silvestre RM. Oral health policies in Brazil. Braz Oral Res. 2009;23(Supl 1):9-16. http://dx.doi.org/10.1590/ S1806-83242009000500003. PMid:19838553.

13. Brasil. Lei ${ }^{\circ} 6.050$, de 24 de maio de 1974. Dispõe sobre a obrigatoriedade da fluoretação das águas em sistemas de abastecimento. Diário Oficial da União, Brasília, 27 de maio de 1975.

14. Brasil. Ministério da Saúde. Coordenação Geral de Saúde Bucal. Guia de recomendações para o uso de fluoretos no Brasil. Brasília; 2009.

15. Brasil. Ministério da Saúde. Secretaria de Atenção à Saúde. Departamento de Atenção Básica. Coordenação Nacional de Saúde Bucal. Diretrizes da Política Nacional de Saúde Bucal. Brasília; 2004.

16. Narvai PC, Frazão P, Roncalli AG, Antunes JLF. Cárie dentária no Brasil: declínio, polarização, iniquidade e exclusão social. Rev Panam Salud Publica. 2006;19(6):385-93. http://dx.doi.org/10.1590/S1020-49892006000600004. PMid:16968593.

17. Antunes JLF, Narvai PC. Políticas de saúde bucal no Brasil e seu impacto sobre as desigualdades em saúde. Rev Saude Publica. 2010;44(2):360-5. http://dx.doi.org/10.1590/S0034-89102010000200018. PMid:20339637.

18. Frazão $P$, Narvai P. Fluoretação da água em cidades brasileiras na primeira década do século XXI. Ver Saúde Pública. 2017;51:47.

19. Brasil. Ministério da Saúde. Portaria no 635, de 26 de dezembro de 1975. Aprova normas e padrões sobre a fluoretação da água de sistemas públicos de abastecimento. Diário Oficial da União, Brasília, 26 de dezembro de 1975 .

20. Brasil. Ministério da Saúde. Portaria no 2.914, de 12 de dezembro de 2011. Dispõe sobre os procedimentos de controle e de vigilância da qualidade da água para consumo humano e seu padrão de potabilidade. Diário Oficial da União, Brasília, 12 de dezembro de 2011.

21. Brasil. Ministério da Saúde. Secretaria de Vigilância em Saúde. Departamento de Vigilância em Saúde Ambiental e Saúde do Trabalhador. Coordenação Geral de Vigilância em Saúde Ambiental. Vigilância da Qualidade da Água para Consumo Humano. Diretriz nacional do plano de amostragem da vigilância da qualidade da água para consumo humano. Brasília: Ministério da Saúde; 2014.

22. Centro Colaborador do Ministério da Saúde em Vigilância da Saúde Bucal. Consenso técnico sobre classificação de águas de abastecimento público segundo o teor de flúor. São Paulo: Faculdade de Saúde Pública, Universidade de São Paulo; 2011.

23. Narvai PC. Cárie dentária e flúor: uma relação do século XX. Cien Saude Colet. 2000;5(2):381-92. http://dx.doi.org/10.1590/S1413-81232000000200011.

24. Silva JS, Val CM, Costa JN, Moura MS, Silva TAE, Sampaio FC. Heterocontrole da fluoretação das águas em três cidades no Piauí, Brasil. Cad Saude Publica. 2007;23(5):1083-8. http://dx.doi.org/10.1590/S0102-311X2007000500010. PMid:17486231.

25. Ramires I, Maia LP, Rigolizzo DS, Lauris JRP, Buzalaf MAR. External control over the fluoridation of the public water supply in Bauru, SP,
Brazil. Rev Saude Publica. 2006;40(5):883-9. http://dx.doi.org/10.1590/ S0034-89102006000600019. PMid:17301911.

26. Panizzi M, Peres MA. Dez anos de heterocontrole da fluoretação de águas em Chapecó, Estado de Santa Catarina, Brasil. Cad Saude Publica. 2008;24(9):2021-31. http://dx.doi.org/10.1590/S0102-311X2008000900008. PMid:18813678.

27. Saliba NA, Moimaz SAS, Saliba O, Barbosa TF. Fluoride content monitoring of the public water supply of the Northwest area of the state of São Paulo, Brazil: 36-month analysis. Rev Odonto Ciênc. 2009;24(4):372-6.

28. Stancari RCA, Dias FL Jr, Freddi FG. Avaliação do processo de fluoretação da água de abastecimento público nos municípios pertencentes ao Grupo de Vigilância Sanitária XV-Bauru, no período de 2002 a 2011. Epidemiol Serv Saude. 2014;23(2):239-48. http://dx.doi.org/10.5123/ S1679-49742014000200005.

29. Coordenação da Região Metropolitana de Curitiba. 2017 [citado em 2017 maio 31]. Disponível em: http://www.comec.pr.gov.br/modules/conteudo/ conteudo.php? conteudo $=123$

30. Centro Colaborador do Ministério da Saúde em Vigilância da Saúde Bucal. Guia de amostragem para vigilância da concentração do fluoreto na água de abastecimento público. São Paulo: Faculdade de Saúde Pública, Universidade de São Paulo; 2014

31. Instituto Brasileiro de Geografia e Estatística. 2017 [citado em 2017 maio 31]. Disponível em: ftp://ftp.ibge.gov.br/Estimativas_de_Populacao/ Estimativas_2016/estimativa_dou_2016_20160913.pdf

32. Centro Colaborador do Ministério da Saúde em Vigilância da Saúde Bucal - CECOL. [citado em 2017 maio 31]. Disponível em: http://www.cecol. fsp.usp.br/cobertura_da_fluoretacao_da_agua/busca_por_municipio

33. Climatempo. Almirante Tamandaré - PR [Internet]. 2017 [citado em 2017 maio 31]. Disponível em: http://www.climatempo.com.br/climatologia/2047/ almirantetamandare-pr

34. Climatempo. Fazenda Rio Grande - PR [Internet]. 2017 [citado em 2017 maio 31]. Disponível em: http://www.climatempo.com.br/climatologia/4249/ fazendariogrande-pr

35. Climatempo. Pinhais - PR [Internet]. 2017 [citado em 2017 maio 31]. Disponível em: http://www.climatempo.com.br/climatologia/4295/pinhaispr

36. Climatempo. Piraquara - PR [Internet]. 2017 [citado em 2017 maio 31]. Disponível em: http://www.climatempo.com.br/climatologia/1327/ piraquara-pr

37. Climatempo. São José dos Pinhais - PR [Internet]. 2017 [citado em 2017 maio 31]. Disponível em: http://www.climatempo.com.br/climatologia/1576/ saojosedospinhais-pr

38. Climatempo. Araucária - PR [Internet]. 2017 [citado em 2017 maio 31]. Disponível em: http://www.climatempo.com.br/climatologia/1302/ araucaria-pr

39. Climatempo. Campo Largo - PR [Internet]. 2017 [citado em 2017 maio 31]. Disponível em: http://www.climatempo.com.br/climatologia/1306/ campolargo-pr

40. Climatempo. Colombo - PR [Internet]. 2017 [citado em 2017 maio 31]. Disponível em: http://www.climatempo.com.br/climatologia/1307/colombopr 
41. Alves RX, Fernandes GF, Razzolini MTP, Frazão P, Marques RAA, Narvai PC. Evolução do acesso à água fluoretada no Estado de São Paulo, Brasil: dos anos 1950 à primeira década do século XXI. Cad Saude Publica. 2012;28(Supl):69-80. http://dx.doi.org/10.1590/S0102-311X2012001300008.

42. Santos CCM, Rodrigues JCS, Lopes MRV, Povinelli RF, Terreri ALM. Avaliação da concentração de flúor nas águas de abastecimento público: estudo retrospectivo e de heterocontrole. Rev Inst Adolfo Lutz. 2012;71(3):507-13.

43. Paredes SO, Sampaio FC, Forte FDS. Heterocontrole da fluoretação das águas de abastecimento público de São Luís, Maranhão. Brazilian Reserch in Pediatric Dentistry and Integrated Clinic. 2014;14(2):129-40.

44. Assaf AV, Teixeira ASC, Silveira FM, Valente MIB, Ditterich RG, Barcelos R Fluoretação da Água e seu Heterocontrole no Município de Nova Friburgo, Rio de Janeiro - Brasil. UNOPAR Cient Ciênc Biol Saúde. 2014;16(3):197201.
45. Peixoto DF, Alencar KP, Peixoto RF, Sousa CFM, Sampaio FC, Forte FDS. External control of fluoridation of public water supplies of the city of Jaguaribara, Ceará, Brazil. Brazilian Journal in Health Promotion. 2012;25(3):271-7.

46. Moimaz SAS, Saliba O, Garbin CAS, Garbin AJI, Sumida DH, Chiba FY, et al. Fluoretação das águas de abastecimento público no município de Araçatuba/SP. Revista Odontológica de Araçatuba. 2012;33(1):54-60.

47. Bergamo ETP, Barbana M, Terada RSS, Cury JA, Fujimaki M. Fluoride concentrations in the water of Maringá, Brazil, considering the benefit/ risk balance of caries and fluorosis. Braz Oral Res. 2015;29(1):1. http:// dx.doi.org/10.1590/1807-3107BOR-2015.vol29.0047. PMid:25760066.

Recebido em: Maio 31, 2017 Aprovado em: Dez. 27, 2017 